\author{
ANNALES \\ POLONICI MATHEMATICI \\ XXIV (1970)
}

\title{
Kovariante Ableitung der Tensordichten
}

von M. KUCHARZEWSKI (Katowice)

Einleitung. In der Arbeit [3] wurde die kovariante Ableitung der Skalare und Dichten definiert und bestimmt. Hier werden die in [3] dargestellten Betrachtungen auf beliebige Tensordichten verallgemeinert. Der erste Paragraph enthält einige Bezeichnungen, die im weiteren nötig sind. In dem zweiten gebe ich die Definition der kovarianten Ableitungen beliebiger Tensordichten an. Dann wird ein Satz über die Existenz und Eindeutigkeit der kovarianten Ableitung bewiesen (\$3). Der vierte Paragraph enthält einige Beziehungen zwischen den kovarianten Ableitungen der Tensordichten verschiedener Valenzen und verschiedener Gewichte. Unter zusätzlichen Voraussetzungen wird die allgemeine Gestalt der kovarianten Ableitung im $\$ 5$ erhalten. Endlich wird gezeigt, wie man die kovariante Ableitung für beliebige Tensordichte erhalten kann, wenn diejenige für Vektoren gegeben ist.

Das kovariante Differential beliebiger Grössen wurde zum ersten Mal von Hlavaty und Schouten [2] axiomatisch definiert (vgl. auch [5] und [6]). Aus den Axiomen für das kovariante Differential kann man die entsprechende Axiome für die kovariante Ableitung erhalten. Hier möchte ich diese Axiome etwas verbessern, insbesondere vereinfachen und genau präzisieren, vor allem aber zeigen, was für Folgen diese nach sich ziehen. Genauere geschichtliche Bemerkungen und Literaturangaben sind in [3] enthalten.

§ 1. Zuerst erinnere ich an die Definition der Tensordichte. Die Tensordichte der Valenz $(p, q)$ ist ein geometrisches Objekt mit $n^{p+q}$ Komponenten im $n$-dimensionalen Raume

$$
\mathfrak{G}_{k_{1} \ldots k_{q}}^{a_{1} \ldots a_{p}}, \quad a_{1}, \ldots, a_{p}, k_{1}, \ldots, k_{q}=1,2, \ldots, n,
$$

die sich bei der Koordinantentransformation

folgendermassen

$$
\xi^{a^{\prime}}=\xi^{a^{\prime}}\left(\xi^{a}\right), \quad a=1,2, \ldots, n,
$$

$$
\mathfrak{G}_{k_{1}^{\prime} \ldots k_{q}^{\prime}}^{a_{1}^{\prime} \ldots a_{p}^{\prime}}=\varphi(J) A_{a_{1}}^{a_{1}^{\prime}} \ldots A_{a_{p}}^{a_{p}^{\prime}} A_{k_{1}^{\prime}}^{k_{1}} \ldots A_{k_{q}^{\prime}}^{k_{q}} \tilde{G}_{k_{1} \ldots k_{q}}^{a_{1} \ldots a_{p}}
$$


transformieren;

$$
A_{a}^{a^{\prime}}=\frac{\partial \xi^{a^{\prime}}}{\partial \xi^{a}}, \quad J=\left|A_{a}^{a^{\prime}}\right| .
$$

Für die $W$-(Weylsche) Dichte mit dem Gewicht $(-\alpha)$ ist $\varphi=|J|^{\sigma}$ und für die $G$-(gewöhnliche) Dichte mit demselben Gewicht ist $\varphi=$ $(\operatorname{sgn} J)|J|^{\alpha}$ (vgl. [3]).

Die Komponenten der Tensordichte (1.1) und ihre Transformationsformel (1.2) werde ich im weiteren in einer kürzeren Form schreiben. Nämlich werde ich mit den ersten grossen lateinischen Buchstaben $A, B, C, D$ die ganzen Folgen von $p$ Indizes $A\left(a_{1}, \ldots, a_{p}\right), B\left(b_{1}, \ldots, b_{p}\right)$, $C\left(c_{1}, \ldots, c_{p}\right), D\left(d_{1}, \ldots, d_{p}\right)$, und mit den grossen lateinischen Buchstaben $K, L, M, N$ aus der Mitte des Alphabets die ganzen Folgen von $q$ Indizes $K\left(k_{1}, \ldots, k_{q}\right), L\left(l_{1}, \ldots, l_{q}\right), M\left(m_{1}, \ldots, m_{q}\right), N\left(n_{1}, \ldots, n_{q}\right)$, bezeichnen. Die $A, B, C, D, K, L, M, N$ sind also die sogenannten Sammelindizes. Mit Hilfe dieser kann die Transformationsformel (1.2) in der Form

$$
\mathfrak{G}_{K^{\prime}}^{A^{\prime}}=\varphi(y) A_{A^{\prime}}^{A^{\prime}} A_{K^{\prime}}^{K} \mathfrak{G}_{K}^{A},
$$

geschrieben werden, wo $A_{A}^{A^{\prime}}$ und $A_{K^{\prime}}^{K}$ durch die Ausdrücke

$$
A_{A}^{A^{\prime}}=A_{a_{1}}^{a_{1}^{\prime}} \ldots A_{a_{p}}^{a_{p}^{\prime}}, \quad A_{K^{\prime}}^{K}=A_{k_{1}^{\prime}}^{k_{1}} \ldots A_{k_{1}^{\prime}}^{k_{1}} \ldots A_{k_{q}^{\prime}}^{k_{q}}
$$

zu ersetzen sind.

§ 2. Die Definition der kovarianten Ableitung der Tensordichte (1.1) hat die folgende Gestalt (vgl. [3]):

Definition 2.1. Als kovariante Ableitung der Tensordichte wird jedes Funktionensystem

$$
F_{M ; x}^{B}=F_{m_{1} \ldots m_{q} ; x}^{b_{1} \ldots b_{p}}, \quad \varkappa=1,2, \ldots, n,
$$

genannt, welches die nachstehenden Bedingungen erfüllt:

$1^{0} F_{M ; x}^{B}$ sind nur von $\mathfrak{G}_{K}^{A}$ und $\mathfrak{G}_{K, \lambda}^{A}\left({ }^{1}\right)$ abhängig:

$$
F_{M ; x}^{B}=F_{M ; x}^{B}\left(\mathfrak{G}_{K}^{A},\left(\mathfrak{F}_{K, \lambda}^{A}\right), \quad \lambda=1,2, \ldots, n ;\right.
$$

$2^{\circ} \quad F_{M ; \varkappa}^{B}$ ist hinsichtlich $\mathfrak{G}_{K}^{A}$ additiv, d.h. für jede zwei Tensordichten ${ }_{1} \mathfrak{G}_{K}^{A},{ }_{2} \mathfrak{F}_{K}^{A}$ derselben Art, Valenz und desselben Gewichtes gilt die Relation

$$
\begin{aligned}
F_{M ; x}^{B}\left({ }_{1} \mathfrak{G}_{K}^{A}+{ }_{2}\left(\mathfrak{F}_{K}^{A},\left({ }_{1} \mathfrak{G}_{K}^{A}+{ }_{2} \mathfrak{F}_{K}^{A}\right), \lambda\right)\right. \\
=F_{M ; x}^{B}\left(_{1} \mathfrak{G}_{K}^{A},{ }_{1}\left(\mathfrak{G}_{K, \lambda}^{A}\right)+F_{M, x}^{B}\left({ }_{2} \mathfrak{F}_{K}^{A},{ }_{2} \mathfrak{G}_{K, \lambda}^{A}\right) .\right.
\end{aligned}
$$

(1) Mit einem Komma wird die partielle und mit einem Semikolon die kovariante Ableitung bezeichnet (vgl. [3]). 
$3^{0}$ Für Produkt von beliebigem Skalar $\sigma$ und der Tensordichte (1.1) gilt die Leibnizsche Regel, d.h.

$$
F_{M ; x}^{B}\left(\sigma \mathfrak{G}_{K}^{A},\left(\sigma \mathfrak{G}_{K}^{A}\right), \lambda\right)=\sigma F_{M ; x}^{B}\left(\mathfrak{G}_{K}^{A}, \mathfrak{G}_{K, \lambda}^{A}\right)+\mathfrak{G}_{M}^{B} \sigma_{, x} .
$$

Ich nehme hier an, daß die kovariante Ableitung eines Skalarfeldes $\sigma$ mit seinem Gradient identisch ist (vgl. [3]): $\sigma_{; x}=\sigma_{, x}$.

$4^{\circ} F_{M ; x}^{B}$ ist eine Tensordichte derselben Art und desselben Gewichtes. wie $\mathfrak{G}_{K}^{A}$, aber der Valenz $(p, q+1)$, d.h. $F_{M ; x}^{B}$ hat die Transformationsformel

$$
F_{M^{\prime} ; x^{\prime}}^{B^{\prime}}=\varphi(J) A_{B}^{B^{\prime}} A_{M}^{M} A_{x^{\prime}}^{*} F_{M ; x}^{B}
$$

Um Missverständnisse $z u$ vermeiden, gebe ich noch einige Erläuterungen der Definition 2.1, insbesondere der Bedingung $1^{\circ}$, an. Alle in dieser Definition auftretenden Objekte sind abstrakte geometrische. Objekte (vgl. [4], S. 24, Definition 8). Die Tensordichte $\mathfrak{G}_{K}^{A}$ ist also das. abstrakte geometrische Objekt mit der Transformationsformel (1.3) und mit der Faser (Fiber, vgl. [4], S. 24, the fibre) $\mathfrak{M}_{0}=R^{N_{0}}, N_{0}=n^{p+q}$. Mit Hilfe dieses Objektes kann ein neues abstraktes geometrisches Objekt. $\left(\mathfrak{F}_{K}^{A},\left(\mathfrak{F}_{K}^{A}, x\right)\right.$ mit der Transformationsformel

$$
\begin{aligned}
& \left(\mathfrak{G}_{K^{\prime}}^{A^{\prime}}=\varphi(J) A_{A}^{A^{\prime}} A_{K^{\prime}}^{K}\left(\mathfrak{G}_{K}^{A},\right.\right.
\end{aligned}
$$

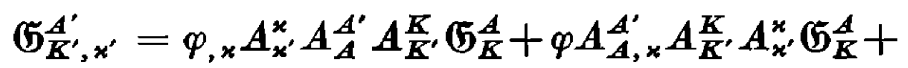

$$
\begin{aligned}
& +\varphi A_{A}^{A^{\prime}} A_{K^{\prime}, x^{\prime}}^{K} \mathfrak{G}_{K}^{A}+\varphi A_{A}^{A^{\prime}} A_{K^{\prime}}^{K} A_{x^{\prime}}^{*} \mathfrak{G}_{K, *}^{A},
\end{aligned}
$$

und mit der Faser $\mathfrak{M}=R^{N}, N=(n+1) n^{p+q}$, gebildet werden.

Mit $\omega_{M \lambda}^{B}=\omega_{m_{1} \ldots m_{q \lambda}}^{b_{1} \ldots b_{p}}$ wird ein abstraktes geometrisches Objekt mit der Transformationsformel

$$
\omega_{M^{\prime} \lambda^{\prime}}^{B^{\prime}}=\varphi(J) A_{B}^{B^{\prime}} A_{M^{\prime}}^{M} A_{\lambda^{\prime}}^{\lambda} \omega_{M \lambda}^{B},
$$

und mit der Faser $\mathfrak{M}_{1}=R^{N_{1}}, N_{1}=n^{p+q+1}$. (Tensordichte der Valenz $(p, q+1)$ mit demselben Gewicht und derselben Art wie $\left(5_{K}^{A}\right)$ bezeichnet.

Das in der Definition 2.1 auftretende Funktionensystem $F_{M \lambda}^{B}$ bildet die Faser $\mathfrak{M}$ in $\mathfrak{M}_{1}$ ab.

Es ist $\mathrm{zu}$ bemerken, dass die kovariante Ableitung keine Differentialkomitante der Tensordichte (1.1) ist. Wir haben nämlich nichts über die Form dieser Ableitung in einem anderen Koordinatensystem vorausgesetzt.

§3. Die Existenz und Eindeutigkeit der kovarianten Ableitung im Sinne der Definition 2.1 wird durch den folgenden Satz bestimmt.

Satz 3.1. Jede kovariante Ableitung der Tensordichten (1.1) hat dieForm

$$
F_{M ; \varkappa}^{B}=\mathfrak{G}_{M, x}^{B}+C_{A M x}^{B K} \mathfrak{G}_{K}^{A}
$$




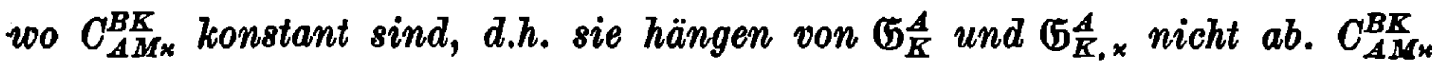
bilden ein geometrisches Objelet, das durch die Transformationsformel

$$
\begin{aligned}
C_{\mathcal{A}^{\prime} M^{\prime} x^{\prime}}^{B^{\prime} K^{\prime}}= & A_{B}^{B^{\prime}} A_{A^{\prime}}^{A} A_{K}^{K^{\prime}} A_{M^{\prime}}^{M} A_{\chi^{\prime}}^{x} C_{A M x}^{B K}+ \\
& +A_{B}^{B^{\prime}} A_{A^{\prime}, x^{\prime}}^{B} \delta_{M^{\prime}}^{K^{\prime}}-A_{K}^{K^{\prime}} A_{M^{\prime}, \alpha^{\prime}}^{K} \delta_{A^{\prime}}^{B^{\prime}}-\alpha \delta_{A^{\prime}}^{B^{\prime}} \delta_{M^{\prime}}^{K^{\prime}} A_{x^{\prime}}^{x} \partial_{\varkappa} \ln |J|,
\end{aligned}
$$

bestimmt ist (vgl. Gheorghiu [1]).

Beweis. Wird in (2.4) für $\sigma$ eine konstante Funktion, eingesetzt, so ist $\sigma_{, *}$ gleich Null und nimmt (2.4) die Form

$$
F_{M ; \varkappa}^{B}\left(\sigma \mathfrak{G}_{K}^{A}, \sigma \mathfrak{G}_{K, \lambda}^{A}\right)=\sigma F_{M ; \varkappa}^{B}\left(\mathfrak{G}_{K}^{A}, \mathfrak{G}_{K, \lambda}^{A}\right),
$$

an, aus deren folgt, dass $F_{M ; x}^{B}$ erster Ordnung homogen ist.

Aus (2.3) und (3.3) ergibt sich weiter, dass $F_{M ; x}^{B}$ linear ist. Sie muss also die Form

$$
F_{M ; x}^{B}=B_{A M}^{B K}\left(\mathfrak{F}_{K, x}^{A}+C_{A M \times}^{B K}\left(\mathfrak{G}_{K}^{A},\right.\right.
$$

haben.

Die linke bzw. rechte Seite von (2.4) kann mit Hilfe von (3.4) in der Form

bzw. in der Form

$$
B_{A M}^{B K} \sigma \mathfrak{G}_{K, x}^{A}+B_{A M}^{B K} \sigma_{, x} \mathfrak{G}_{K}^{A}+C_{A M x}^{B K} \sigma \mathfrak{G}_{K}^{A}
$$

$$
\sigma B_{A M}^{B K}\left(\mathfrak{G}_{K, x}^{A}+\sigma C_{A M x}^{B K}\left(\mathfrak{G}_{K}^{A}+\mathfrak{G}_{M}^{B} \sigma_{, x}\right.\right.
$$

geschrieben werden. Durch Vergleichen von (3.5) und (3.6) ergibt sich die Relation

$$
B_{A M}^{B K} \sigma_{, x} \mathfrak{\varpi}_{K}^{A}=\mathfrak{\varpi}_{M}^{B} \sigma_{, x}
$$

die in der Form

$$
\left(B_{A M}^{B K}-\delta_{A}^{B} \delta_{M}^{K}\right) \sigma_{, x}\left(\mathfrak{G}_{K}^{A}=0,\right.
$$

dargestellt werden kann. Da aber $\mathfrak{G}_{K}^{A}$ und $\sigma_{, x}$ beliebig sind, aus (3.8) erhält man

$$
B_{A M}^{B K}=\delta_{A}^{B} \delta_{M}^{K}
$$

Wird (3.9) in (3.4) eingesetzt, so nimmt diese die im Satz 3.1 erforderte Form (3.1) an.

Jetzt wird die Transformationsformel (3.2) von $C_{A M x}^{B K}$ abgeleitet. $\mathrm{Zu}$ diesem $\mathrm{Zw}_{\mathrm{w}}$ cke schreibe ich (3.1) in einem anderen Koordinatensystem

$$
F_{M^{\prime} ; \boldsymbol{x}^{\prime}}^{B^{\prime}}=\mathfrak{G}_{M^{\prime}, \boldsymbol{x}^{\prime}}^{B^{\prime}}+C_{\boldsymbol{A}^{\prime} \mathbf{M}^{\prime} \mathbf{x}^{\prime}}^{B^{\prime} \boldsymbol{G}_{K^{\prime}}^{A^{\prime}}}
$$

Mit Hilfe der Transformationsformel (1.3) von $\mathfrak{5}_{M}^{B}$ kann man die Ableitungen $\mathfrak{G}_{M^{\prime}, \boldsymbol{x}^{\prime}}^{B^{\prime}}$ der Tensordichte im neuen Koordinatensystem berechnen:

$$
\begin{aligned}
\mathfrak{G}_{M^{\prime}, x^{\prime}}^{B^{\prime}}=\varphi_{, x^{\prime}} A_{B}^{B^{\prime}} A_{M^{\prime}}^{M} \mathfrak{G}_{M}^{B}+\varphi A_{B, x^{\prime}}^{B^{\prime}} A_{M^{\prime}}^{M} \mathfrak{G}_{M}^{B}+ \\
+\varphi A_{B}^{B^{\prime}} A_{M^{\prime}, x^{\prime}}^{M} \mathfrak{G}_{M}^{B}+\varphi A_{B}^{B^{\prime}} A_{M^{\prime}}^{M} \cdot A_{\mathcal{x}^{\prime}}^{\boldsymbol{x}} \mathfrak{G}_{M, x}^{B}
\end{aligned}
$$


Durch Einsetzten von (3.11), (1.3), (2.5) in (3.10) ergibt sich

(3.12) $\varphi A_{B}^{B^{\prime}} A_{M^{\prime}}^{M} A_{x^{\prime}}^{\chi} C_{A M x}^{B K}\left(5_{K}^{A}\right.$

$=\varphi_{, x^{\prime}} A_{B}^{B^{\prime}} A_{M^{\prime}}^{M} \mathfrak{G}_{M}^{B}+\varphi A_{B, x^{\prime}}^{B^{\prime}} A_{M^{\prime}}^{M}, \mathfrak{G}_{M}^{B}+\varphi A_{B}^{B^{\prime}} A_{M^{\prime} x^{\prime}}^{M} \mathfrak{G}_{M}^{B}+C_{A^{\prime} M^{\prime} x^{\prime}}^{B^{\prime} K^{\prime}} \varphi A_{A}^{A^{\prime}} A_{K^{\prime}}^{K} \mathfrak{G}_{K}^{A}$.

Die Funktion $\varphi$ erfüllt die in [3] bewiesene Gleichung

$$
\varphi^{\prime}(\varrho)=\frac{a \varphi(\varrho)}{\varrho}
$$

Die Ableitung $\varphi_{, x^{\prime}}$ ist also gleich

$$
\varphi_{, x^{\prime}}=\varphi^{\prime}(J) J_{, x^{\prime}}=\frac{a \varphi(J)}{J} J_{, x^{\prime}}=\alpha \varphi \partial_{x^{\prime}} \ln |J| .
$$

Jetzt setzen wir (3.14) in (3.12) ein und vernachlässigen die $\varphi$ und $\boldsymbol{G}_{K}^{A}$, weil diese beliebige Werte annehmen können. Dann erhält man die Relation

$$
\begin{array}{r}
A_{B}^{B^{\prime}} A_{M^{\prime}}^{M} A_{\chi^{\prime}}^{x} C_{A M x}^{B K}=a \partial_{x^{\prime}} \ln |J| \cdot A_{B}^{B^{\prime}} A_{M^{\prime}}^{M} \delta_{A}^{B} \delta_{M}^{K}+A_{B, x^{\prime}}^{B^{\prime}} A_{M^{\prime}}^{M} \delta_{A}^{B} \delta_{M}^{K}+ \\
+A_{B^{\prime}}^{B} A_{M^{\prime}, x^{\prime}}^{M} \delta_{A}^{B} \delta_{M}^{K}+C_{A^{\prime} M^{\prime} x^{\prime}}^{B^{\prime} A_{A}^{A^{\prime}} A_{K^{\prime}}^{K}},
\end{array}
$$

die mit (3.2) äquivalent ist. Auf diese Weise ist der Satz 3.1 vollständig bewiesen.

\$ 4. Die in der Formel für die kovariante Ableitung (3.1) auftretenden Konstanten $C_{\boldsymbol{A M x}}^{B K}$ können im allgemeinen von den Gewichten, Valenzen und Arten der betrachteten Tensordichten abhängen. Man kann also $C_{A M x}^{B K}$ als Funktionen von $a$ und $\varepsilon$,

$$
C_{A M x}^{B K}=C_{A M x}^{B K}(\alpha, \varepsilon)
$$

darstellen, wo $(-a)$ das Gewicht der Tensordichte bedeutet und $\varepsilon$ für $W$-Dichten gleich 1 und für $G$-Dichten gleich (-1) ist (vgl. [3]). Die Abhängigkeit von der Valenz wird durch die Anzahl und Art der Indizes ausgedrückt.

Jetzt möchte ich eine Beziehung für die Funktionen (4.1) ableiten. Za diesem Zwecke muss man die Leibnizsche Regel für Produkte zweier beliebiger Tensordichten voraussetzen.

Es seien also

$$
{ }_{1} \mathfrak{5}_{K}^{A},{ }_{2} \mathbf{5}_{A}^{\Gamma}
$$

zwei Tensordichten. Die Indizes $A, K$ sind schon im $\S 1$ definiert und $\Gamma, \Delta, \Lambda, \Omega$ sind durch die Bezeichnungen: $\Gamma\left(\gamma_{1}, \ldots, \gamma_{\ell}\right), \Delta\left(\delta_{1}, \ldots, \delta_{\ell}\right)$, $\Lambda\left(\lambda_{1}, \ldots, \lambda_{\sigma}\right), \Omega\left(\omega_{1}, \ldots, \omega_{\sigma}\right), \gamma_{1} \ldots \gamma_{e}, \delta_{1} \ldots \delta_{e}, \lambda_{1} \ldots \lambda_{\sigma}, \omega_{1} \ldots \omega_{\sigma}=1,2, \ldots$ $\ldots, n$, erklärt. 
Der Transformationsformel (1.2) gemäss ist das Gewicht von ${ }_{1} \boldsymbol{F}_{K}^{A}$ gleich $(-\alpha)$. Es sei $(-\beta)$ das Gewicht von ${ }_{2} \boldsymbol{5}_{\Lambda}^{T}$. Das allgemeine Produkt von ${ }_{1}\left(\mathfrak{G}_{K}^{A}\right.$ und ${ }_{2} \mathfrak{G}_{A}^{\Gamma}$ ist auch eine Tensordichte von der Valenz $(p+\varrho, q+\sigma)$ mit dem Gewicht $-(\alpha+\beta)$ und entsprechender Art, die von den Arten ${ }_{1} \mathfrak{F}_{K}^{A}$ und ${ }_{2} \mathfrak{G}_{A}^{\Gamma}$ abhängig ist. Aus Satz 3.1 folgt, dass die kovariante Ableitungen der Tensordichten ${ }_{1} \mathfrak{G}_{K}^{A},{ }_{2} \mathfrak{G}_{A}^{\Gamma}$ und ihres Produktes ${ }_{1} \mathfrak{G}_{K}^{A}{ }_{2} \mathfrak{G}_{A}^{\Gamma}$ die folgenden Formen

$$
\begin{aligned}
& F_{K ; \varkappa}^{A}={ }_{1}\left(\mathfrak{F}_{K, \varkappa}^{A}+C_{B K \varkappa}^{A M}\left(\alpha, \varepsilon_{1}\right)_{1} \mathfrak{b}_{M}^{B},\right. \\
& F_{\Lambda ; x}^{\Gamma}={ }_{2} \mathfrak{G}_{\Lambda, x}^{\Gamma}+C_{\Delta A x}^{\Gamma \Omega}\left(\beta, \varepsilon_{2}\right)_{2}\left(\mathfrak{G}_{\Omega}^{A},\right. \\
& F_{K A ; x}^{A \Gamma}=\left({ }_{1} \mathfrak{G}_{K 2}^{A}\left(\mathfrak{G}_{A}^{T}\right)_{, x}+C_{B \Delta K \Lambda x}^{A \Gamma M \Omega}\left(\alpha+\beta, \varepsilon_{2} \varepsilon_{1}\right)_{1}\left(\mathfrak{G}_{M 2}^{B} \mathfrak{G}_{\Omega}^{A},\right.\right.
\end{aligned}
$$

haben.

Die in diesem Paragraphen vorausgesetzte Leibnizsche Produktregel kann mit Hilfe der kovarianten Ableitungen (4.3), (4.4), (4.5) folgendermassen ausgedrückt werden:

$$
F_{K A ; x}^{A \Gamma}={ }_{1} \mathfrak{G}_{K}^{A} F_{\Lambda ; \varkappa}^{\Gamma}+{ }_{2} \mathfrak{G}_{\Lambda}^{\Gamma} F_{K ; x}^{A} .
$$

Durch Einsetzen der rechten Seiten von (4.3), (4.4) und (4.5) in (4.6), ergibt sich die Gleichung

$$
C_{B \Delta K A x}^{A \Gamma M \Omega}\left(\alpha+\beta, \varepsilon_{2} \varepsilon_{1}\right)=\delta_{B}^{A} \delta_{K}^{M} C_{\Delta A x}^{\Gamma \Omega}\left(\beta_{1} \varepsilon_{2}\right)+\delta_{\Delta}^{\Gamma} \delta_{\Lambda}^{\Omega} C_{B K x}^{A M}\left(a, \varepsilon_{1}\right){ }^{(1)} .
$$

Dieses Ergebnis wird im nachstehenden Satz zusammengefasst.

SaTz. 4.1. Die kovarianten Ableitungen der Tensordichten erfüllen dann und nur dann die Leibnizsche Produktregel, wenn die Relation (4.7) erfüllt ist.

Wie das aus (3.1) folgt, ist die kovariante Ableitung der Tensordichte $\mathfrak{G}_{K}^{A}$ durch das Objekt $C_{A M x}^{B K}$ vollständig bestimmt. Darum wird es der Parameter der kovarianten Ableitung bzw. der linearen Übertragung genannt.

§5. Jetzt werden einige Aussagen über die Form der Parameter $C_{A M x}^{B K}(a, \varepsilon)$ abgeleitet. Sie sind Verallgemeinerungen der in [3] bewiesenen Sätze über die Parameter der kovarianten Ableitung der Dichten.

Wir betrachten zuerst den Spezialfall der Relation (4.7), der entsteht, wenn man als ${ }_{2}\left(5_{\Lambda}^{\Gamma}={ }_{2}(5\right.$ eine Dichte, d.h. Tensordichte der Valenz $(0,0)$,

(1) Die Formel (4.7) wurde nur für die Parameter dieser Tensordichten $\boldsymbol{G}_{K A}^{A} \boldsymbol{A}$ abgeleitet, die als Produkt von ${ }_{1} \mathfrak{G}_{K}^{A}$ und ${ }_{2} \mathfrak{G}_{A}^{T}$ dargestellt werden können. Da aber $\sigma_{B \Delta K \Delta x}^{A \Gamma M S}$ von $G_{K A}^{A \Gamma}$ nicht abhängen (Satz 3.1) ist diese Formel für jede Tensordichte der Valenz $(\varrho+\varrho, q+\sigma)$ mit dem Gewicht $-(\alpha+\beta)$ gültig. Auf diese Weise sind alle anderen Formel dergleichen Art in dieser Arbeit zu verstehen. 
annimmt. Dann treten die griechischen Indizes in (4.7) nicht auf. Die Formel (4.7) hat also die Form

$$
C_{B K \varkappa}^{A M}\left(\alpha+\beta, \varepsilon_{2} \varepsilon_{1}\right)=\delta_{B}^{A} \delta_{K}^{M} C_{\varkappa}\left(\beta, \varepsilon_{2}\right)+C_{B K \varkappa}^{A M}\left(\alpha, \varepsilon_{1}\right) .
$$

Aus [3] (Satz 5.1) folgt, dass $C_{x}$ von der Art der Dichte unabhängig ist, wenn die Leibnizsche Produktregel für die Dichten gilt. Mann kann also in (5.1),

$$
C_{\varkappa}\left(\beta, \varepsilon_{2}\right)=C_{\varkappa}(\beta),
$$

einsetzen. Dann erhalten wir die Relation

$$
C_{B K_{x}}^{A M}\left(\alpha+\beta, \varepsilon_{2} \varepsilon_{1}\right)=\delta_{B}^{A} \delta_{K}^{M} C_{x}(\beta)+C_{B K^{x}}^{A M}\left(\alpha, \varepsilon_{1}\right) .
$$

Wird $\varepsilon_{1}=1$ und $\beta=0$ in (5.3) eingesetzt, sor ergibt sich, dass die $C_{B K x}^{A M}$ von $\varepsilon$ nicht abhängen (die rechte Seite enthält $\varepsilon_{2}$ und die linke hängt von $\varepsilon_{2}$, nicht ab). Auf diese Weise erhalten wir den

HuFssatz 5.1. Die Parameter der kovarianten Ableitung hängen von der Art der Tensordichte nicht ab, wenn die Leibnizsche Regel für Produkte der Dichten und Tensordichten gilt.

Die Relation (5.1) kann jetzt in der Form

$$
C_{B K \varkappa}^{A M}(\alpha+\beta)=\delta_{B}^{A} \delta_{K}^{M} C_{\varkappa}(\beta)+C_{B K \varkappa}^{A M}(\alpha),
$$

geschrieben werden, aus der als Spezialfall erhalten wir

$$
C_{B K \varkappa}^{A M}(\beta)=\delta_{B}^{A} \delta_{K}^{M} C_{\varkappa}(\beta)+C_{B K \varkappa}^{A M}(0) .
$$

Daraus folgt

Satz 5.1. Genügt die kovariante Ableitung der Leibnizschen Produktregel, so sind ihre Parameter eindeutig durch diejenigen für Dichten $C_{x}(\beta)$ und Tensoren $C_{B K \varkappa}^{A M}(0)$, durch die Formel (5.5), eindeutig bestimmt. Die $C_{\varkappa}(\beta)$ bzw. $C_{B K \boldsymbol{A}}^{A M}$ erfüllen die Gleichung

bzw.

$$
C_{x}(a+\beta)=C_{x}(\alpha)+C_{x}(\beta),
$$

$$
C_{B \Delta K A \varkappa}^{A \Gamma M \Omega}(0)=\delta_{B}^{A} \delta_{K}^{M} C_{\Delta A \varkappa}^{\Gamma \Omega}(0)+\delta_{\Delta}^{\Gamma} \delta_{A}^{\Omega} C_{B K \varkappa}^{A M}(0)
$$

§ 6. Um also die Parameter der kovarianten Ableitung $C_{B K x}^{A M}$ für beliebige Tensordichten zu bilden, genügt es nur diejenigen für Dichten $C_{x}(\beta)$ und Tensoren $C_{B K x}^{A M}(0)$ anzugeben. Diese können nicht beliebig sein, sondern müssen die Gleichungen (5.6) und (5.7) erfüllen. Jetzt zeige ich, wie die Parameter der kovarianten Ableitung beliebiger Tensordichten $C_{B K x}^{A M}(\alpha)$ eindeutig gebildet werden können, wenn diejenigen für kontravariante Vektoren $\Gamma_{b x}^{a}$ angegeben sind. 
Es seien

bzw.

$$
\Gamma_{b x}^{a}=\Gamma_{b x}^{a}(0)
$$

$$
\Phi_{m x}^{k}=\Phi_{m x}^{k}(0),
$$

die Parameter der kovarianten Ableitung für kontravariante bzw. kovariante Vektoren. Unter der Voraussetzung, dass die Leibnizsche Produktregel erfüllt ist, haben die Parameter der kovarianten Ableitung für Tensoren der Valenz $(p, 0),(0, q)$ und für die Dichten mit dem Gewicht $(-1)$ die folgenden Formen:

$$
\begin{aligned}
& C_{B \ltimes}^{A}(0)=\sum_{i=1}^{p} \delta_{b_{1} \cdots}^{a_{1} \cdots} \delta_{b_{i-1}}^{a_{i-1}} \Gamma_{b_{i^{\kappa}}}^{a_{i}} \delta_{b_{i+1} \cdots}^{a_{i+1} \cdots} \delta_{b_{p}}^{a_{p}} \\
& C_{M \times}^{K}(0)=\sum_{j=1}^{q} \delta_{m_{1} \ldots}^{k_{1} \ldots} \delta_{m_{j-1}}^{k_{j-1}} \Phi_{m_{j \times}}^{k_{j}} \delta_{m_{j+1} \ldots}^{k_{j+1} \ldots} \delta_{m_{q}}^{k_{q}}, \\
& \Phi_{*}=C_{x}(1)=\Gamma_{a x}^{a},
\end{aligned}
$$

wo $\Gamma_{b x}^{a}$ bzw. $\Phi_{m x}^{k}$ die angegebenen Parameter (6.1) bzw. (6.2) bedenten. Die Formel (6.3) wird mit Hilfe der vollständigen Induktion hinsichtlich $p$ bewiesen.

Für $p=2$ geht (6.2) in die Formel

$$
C_{b_{1} b_{2}}^{a_{1} a_{2}}=\delta_{b_{1}}^{a_{1}} \Gamma_{b_{2 x}}^{a_{2}}+\delta_{b_{2}}^{a_{2}} \Gamma_{b_{1}{ }^{x}}^{a_{1}},
$$

über. Diese ergibt sich, wenn wir in (5.7) $A=\left(a_{1}\right), B=\left(b_{1}\right), \dot{\Gamma}=\left(a_{2}\right)$, $\Delta=\left(b_{2}\right)$ einsetzen (die Indizes $M, K, \Lambda, \Omega$, treten in (6.3) nicht auf). Für $p=2$ ist also die Formel (6.3) erfïllt. Wir nehmen jetzt an, dass (6.3) gilt und wir werden zeigen, dass diese, wenn wir $p$ durch $p+1$ ersetzen, richtig bleibt. $\mathrm{Zu}$ diesem Zwecke setzen wir die folgenden Werte für Indizes

$$
\boldsymbol{A}=\left(a_{1}, \ldots, a_{p}\right), \quad B=\left(b_{1}, \ldots, b_{p}\right), \quad \Gamma=\left(a_{p+1}\right), \quad \Delta=\left(b_{p+1}\right),
$$

in (5.7) ein. Dann nimmt (5.7) die Form

$$
C_{B \Delta \varkappa}^{A} \Gamma=\delta_{B}^{A} \Gamma_{b p+1^{\varkappa}}^{a_{p+1}}+\delta_{b_{p+1}}^{a_{p+1}} C_{B \varkappa}^{A}
$$

an. Wegen der Induktionsvoraussetzung haben $C_{B_{k}}^{A}$ die Form (6.3). Wenn wir also für diese die rechte Seite von (6.3) in (6.7) einsetzen, so erhält man die Formel (6.3) für $p+1$.

Ganz analog kann die Formel (6.4) bewiesen werden.

Um (6.5) zu beweisen, berechnen wir die kovariante Ableitung der Dichte $\mathfrak{M}$, die aus $n$ kontravarianten Vektoren $v, \ldots, v$ mittels der Gleichung

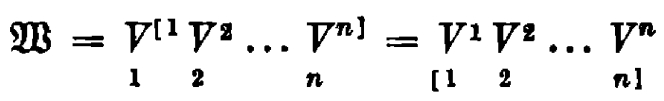


gebildet ist. Da $\mathfrak{W}$ eine gewöhnliche Dichte mit dem Gewicht ( -1$)$ ist muss ihre kovariante Ableitung (Satz 4.1, [3]) die Form

$$
\mathfrak{W}_{; x}=\mathfrak{W}_{, x}+\Phi_{x} \mathfrak{W}
$$

haben. Anderseits kann man die kovariante Ableitung von $\mathfrak{W}$ mit Hilfe von (6.8) und der Leibnizschen Produktregel folgendermassen bestimmen:

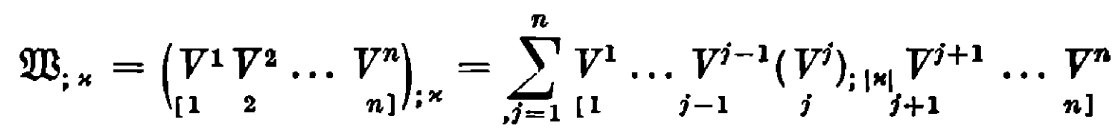

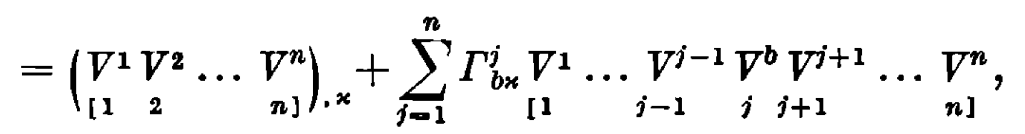

$$
\mathfrak{W}_{; \varkappa}=\mathfrak{W}_{, x}+\Gamma_{b x}^{b} \mathfrak{W} \text {. }
$$

Durch Vergleichen der rechten Seiten von (6.9) und von (6.10), da $\mathfrak{B}$ beliebige Werten annehmen kann, erhalten wir (6.5).

Jetzt bestimmen wir den Zusammenhang zwischen den Parametern $\Gamma_{b x}^{a}$ und $\Phi_{-m x}^{k}$.

Huffssatz 6.1. Erfüllt die kovariante Ableitung die Leibnizsche Regel für die Überschiebungen der ko- und kontravarianten Veltoren, d.h. gilt die Relation

$$
\left(V^{a} U_{a}\right)_{; x}=\nabla_{; x}^{a} U_{a}+V^{a}\left(U_{a}\right)_{; x},
$$

für beliebige ko- und kontravariante Vektoren $\nabla^{a}, U_{a}$, so können $\Phi_{m \times}^{k}$ durch $\Gamma_{b x}^{a}$ mittels der Formel

$$
\Phi_{m x}^{k}=-\Gamma_{m x}^{k}
$$

ausgedrückt werden.

Beweis. Da die Uberschiebung $V^{a} U_{a}$ ein Skalar darstellt, ist ihre kovariante Ableitung mit dem Gradient identisch ([3], Satz 2.1),

$$
\left(V^{a} U_{a}\right)_{; \varkappa}=\left(V^{a} U_{a}\right)_{, x} .
$$

Die Beziehung (6.12) wird erhalten, wenn man die linke Seite von (6.13) durch die rechte von (6.11) ersetzt und die dort auftretenden kovarianten Ableitungen durch entsprechendene Formel (3.1) eliminiert.

Mit Hilfe von (6.12) können die Parameter (6.4) in der Form

$$
C_{M \times}^{K}=-\sum_{j=1}^{q} \delta_{m_{1}}^{k_{1}} \ldots \delta_{m_{j-1}}^{k_{j-1}} \Gamma_{m_{j} \times}^{k_{j}} \delta_{m_{j+1}}^{k_{j+1}} \ldots \delta_{m_{2}}^{k_{2}},
$$

geschrieben werden.

Die soeben erhaltenen Resultate fassen wir im nachstehenden Satz zusammen, der die allgemeine Gestalt der Parameter der kovarianten Ableitung für beliebige Tensordichte gibt. 
Satz 6.1. Sind die Leibnizsche Regel für Produkte beliebiger Tensordichten und für die Überschiebungen der ko- und kontravarianten Velktoren erfïllt, so sind die Parameter der kovarianten Ableitung beliebiger Tensordichten $C_{B M x}^{A K}(a)$ durch diejenigen für kontravariante Vektoren $\Gamma_{b x}^{a}(0)$ und diejenigen für Dichten. $C_{*}(a)$ eindeutig bestimmt. Sie haben die Form

$$
C_{B M \times}^{A K}(a)=\delta_{B}^{A} C_{M \varkappa}^{K}(0)+\delta_{M}^{K} C_{B \times}^{A}(0)+C_{x}(\alpha) \delta_{B}^{A} \delta_{M}^{K},
$$

wo $C_{B \times}^{A}, C_{M \varkappa}^{K}$ und $C_{*}(a)$ die Parameter der kovarianten Ableitung für kovariante (6.14) bzw. kontravariante Tensoren (6.3) und $C_{*}(a)$ diejenigen für Dichten bedeuten.

Die Formel (6.15) folgt ohne weiteres aus (5.5) und (5.7), wenn wir die Indizes $\Gamma, \Delta, K, M$, in. (5.7) weglassen und $\Omega, \Lambda$, durch $K, M$, ersetzen.

Sind die Parameter $C_{x}(\alpha)$ der kovarianten Ableitung der Dichten messbar, so haben șie die Form ([3], Satz 5.2)

$$
C_{\varkappa}(\alpha)=a C_{x}(1)=a \Phi_{\star} .
$$

Wegen (6.5) erhalten wir daraus

$$
C_{x}(a)=\alpha \Gamma_{a x}^{a} .
$$

Dann nimmt die Formel (6.15) die folgende Gestalt

$$
C_{B M x}^{A K}(a)=\delta_{B}^{A} C_{M \varkappa}^{K}(0)+\delta_{M}^{K} C_{B \varkappa}^{A}(0)+\alpha \Gamma_{a x}^{a} \delta_{B}^{A} \delta_{M}^{K},
$$

an. In diesem Falle kann der Satz 6.1 so dargestellt werden:

SATz 6.2. Sind die Voraussetzungen des Satzes 6.1 erfïllt und sind die Parameter $C_{*}(\alpha)$ messbar, so können die Parameter $C_{B M *}^{A K}(a)$ der kovarianten Ableitung beliebiger Tensordichten durch diejenigen für kontravariante Veltoren $\Gamma_{b x}^{a}$ mit Hilfe der Formel (6.18) eindeutig bestimmt werden.

\section{Literaturnachweis}

[1] O. E. Gheorghiu, Determinarea derivatei covariante a pseudotensorilor, Bul. Sti. Teh. Institut Polit. Tomişoara 1 (15), (1956), S. 27-30.

[2] V. Hlavaty und J. A. Schouten, Zur Theorie der allgemeinen linearen Übertragung, Math. Z. 30 (1929), S. 414-432.

[3] M. Kucharzewski, Kovariante Ableitung der Skalare und Dichten, Prace Mat. U. S. Katowice 1 (1969), S. 61-70.

[4] - and M. Kuozma, Basic concepts of the theory of geometric objects, Rozprawy Mat. 43, Warszawa 1964.

[5] J. A. Schouten, Ricci Oalculus, Berlin-Göttingen-Heidelberg 1954.

[6] - und D. J. Struik, Einführung in die neueren Methoden der Differentialgeometrie I, Gronningen 1935. 